\title{
Design and Improvement of the Automatic Control System of Pig Feed Production Line based on the Internet of Things and Innovative Thinking Mode
}

\author{
Bai Yan \\ Engineering Training Center, Beihua University, Jilin 132021
}

Keywords: Selenium; feed production line; T-S model; internet of things

\begin{abstract}
This paper mainly focuses on the feed production line of a certain feed company in Jilin, the design of a segmented pig feed line under the concept of innovative thinking, and the large data technology, Internet of things technology applied to the production line, the overall design of the segmented production line is completed. The test results show that the system has good stability, innovation and functionality.
\end{abstract}

\section{Introduction}

This paper takes the production line of a feed enterprise as the research object, it presents a design of intelligent automatic production control system for selenium enriched pig feed based on Internet of things and innovative thinking, it will be the big data technology and networking technology applied to the production line, through the cloud computing method on line data acquisition and processing, and through the analysis of intelligent control strategy to improve the control precision and control effect [1].

A new concept is presented in this paper-selenium, $t$ is a necessary trace element for the human body. Scientific research shows that the content of selenium is closely related to the incidence of cancer, Therefore, the scientific community called selenium "the king of anti-cancer"'",It can not only eliminate the toxin in the body, prevent blood clots, remove cholesterol, enhance the body's immunity and can prevent cardiovascular disease, Keshan disease and Beck disease, do protective effects of detoxification. In this paper, selenium was added to feed processing. Let hormones, pork away from the table, so that functional, green, healthy food slowly immersed in the heart [1][2].

\section{Hardware Structure Design of Automatic Control System for Production Line}

The production process of selenium enriched pig feed mainly includes raw material storage, raw material crushing, batching processing, mixed allocation, granulating cooling, finished product packaging, etc..1. Raw material storage: when raw materials are stored, different raw materials receiving and cleaning processes should be adopted due to different raw material varieties and packaging methods, such as easy handling of grain and good liquidity, generally exist in the silo of the plant. Powder class, such as cake, fish meal and other poor liquidity is generally stored in the warehouse. Liquids, such as oils, amino acids, etc. are stored in storage tanks. Drugs and additives, such as trace elements need special storage, when the ingredients into the production equipment in accordance with the proportion. Clean raw materials to protect the equipment at the same time in order to guarantee the quality of feed, raw materials will be collected in the course of the rope, stones, iron and other impurities removed. In this paper, the vibrating equipment is equipped with a permanent magnetic cylinder, and the magnetic small impurities are adsorbed.2. Grinding: grinding the raw materials into powder by grinding machine, and then transport different raw materials to the batching bin through the conveyor. 3. Ingredients: ingredients is the core of feed production, the use of fully automated process, formula ratio has been programmed, and add automatic proportioning bin of the raw materials added after precision measurement of various sensors, to ensure data after the completion of the proportion of ingredients, said the door opened automatically, the configuration of the feed input into a mixing machine. 4. Mixing: evenly mixing raw materials and auxiliary materials in the batching process. 5. Granulation: the mixed powder feed is processed into 
granules, and the process includes screening, quenching and tempering, granulation, cooling, crushing and sieving. 6. packaging: automatic packing machine weighing, bagging and sealing the finished product [3].

Selenium granule pig feed due to the application object is a process for the production of piglets, so the pellet grinding process requirements are very high, the traditional feed crushing device for crushing efficiency is low, only the production of fixed size feed, when the production of a fine grain feed, the crushing effect is not ideal, have to wait out material and then into crushing device. By two feeding, very inconvenient, so the traditional feed grinding device needs to be improved. Selenium granule pig feed improved crushing device in the first and second motors driven by the first crushing blade and second crushing blades rotate in the opposite direction, the interaction in the first blade and the second blade broken, improve the crushing efficiency; crushing barrel shell is provided with a groove, crushing bucket and sieve thread the connection, by changing the different specifications of the filter screen of pig feed to produce different particle sizes; a baffle installation wall broken barrels, qualified feed through the filter sieve outflow from the outlet, unqualified in crushing barrel, was brought to the top of the first baffle broken blade down again to avoid crushing two feeding; the utility model has the advantages of high crushing efficiency, no dead angle, crushing particle size adjustable, suitable for large-scale promotion[3].The mechanical structure of selenium enriched pig feed grinding device is shown in Figure 1.

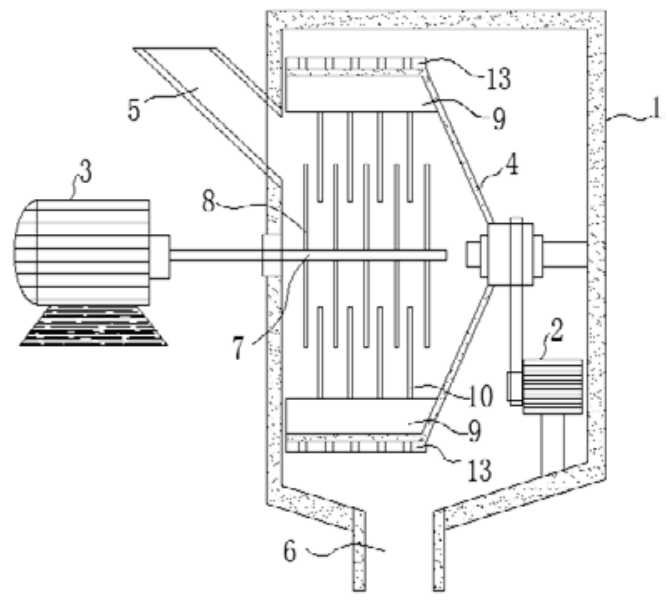

In the diagram: 1 shell, 2 first motor, 3 second motor, 4 broken barrel, 5 oblique feed port, 6 outlet, 7 crushing shaft, 8 first broken blade, 9 baffle, 10 second broken blade, 11 slot, 12 thread hole, 13 filter screen

Fig. 1 mechanical structure drawing of selenium enriched pig feed grinding device

\section{Electrical System Design of Automatic Control System for Production Process}

The automatic control system of selenium enriched pig feed production is composed of weight sensor, weighing instrument, industrial control computer, touch screen, analog screen, SIEMENS PLC and other electrical equipment [4].

The production process monitoring and management of the computer to complete the feed processing, the proportion of raw materials and ingredients, formulation, time parameter setting, the workshop equipment adopts touch screen provides a good human-computer interface for operation management personnel, the machine adopts SIEMENS PLC, through the general line and Ethernet communication field instrument, transducer, sensor. The frequency converter completes the speed control of feeding equipment. At the same time, the control system to achieve the best control effect, in the process of T-S model and PID organic combination, in the sensor detection part and material weighing process to improve the stability of the role.

T-S model is an effective model to solve nonlinear problems, can describe the nonlinear dynamic characteristics of the system, so it has been widely used, combined with rich selenium for particles of pig feed production process automatic control can get good control effect in the control system of T-S model and PID. TS-PID is more effective than PID in parameter adjustment. Suppose there is 
$\mathrm{M}$ rule, then ${ }^{i}$ the rule is that the rule is:

$$
\begin{gathered}
R^{i}: \text { if } h_{3} \text { is } A^{i} \text {, then } \\
k_{P}=\boldsymbol{k}_{p^{i}}^{i}, k_{i}=\boldsymbol{k}_{i}^{i}, k_{d}=\boldsymbol{k}_{d^{\prime}}^{i},(i=1,2 \ldots) \\
K_{P}=\frac{\sum_{i=1}^{n} w_{A^{\prime}} \boldsymbol{k}_{p}^{i}}{\sum_{i=1}^{n} w_{A^{\prime}}} ; K_{i}=\frac{\sum_{i=1}^{n} w_{A^{\prime}} \cdot \boldsymbol{k}_{i}^{i}}{\sum_{i=1}^{n} w_{A^{\prime}}} ; K_{d}=\frac{\sum_{i=1}^{n} w_{A^{\prime}} \boldsymbol{k}_{d}^{i}}{\sum_{i=1}^{n} w_{A^{\prime}}}
\end{gathered}
$$

Among them, the fuzzy linguistic rule is applicable; A is fuzzy subset; the state of the controlled object is the parameter value of the output variables PID, $K_{p}, K_{i}, K_{d}$. The simulation model is built as shown in figure 2.
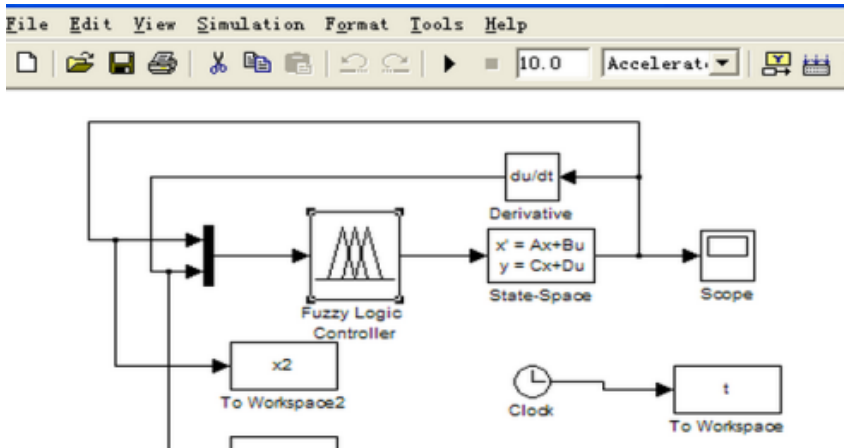

Fig. 2 simulation model

\section{Software Design of Automatic Control System for Production Process}

Internet of things=Internet plus sensor, it leads the development of industry. The general production industry to the intelligent industry, so that the development of the industry into the wisdom of the tentacles, so that the feed industry from a single to multiple integration. The big data is a large amount of data acquisition, rapid analysis from a large number of data information and draw the law, suggesting that the development of the industry information, is our tool to solve the problem. The combination of Internet of things and big data should be used in feed production industry, improve the top-level design, unify the system platform, realize the data sharing, make the production system more secure, professional and unified. In the joint perception layer, we should complete the perception of environment perception, personnel identity perception, location awareness, behavior perception, raw materials and ingredients information. Application layer should have authority management, equipment management, report management, personnel management, operation and maintenance management, security management, communication management, account management, network management, data management. Interface extension layer has a variety of application interfaces, in the platform application services to complete the big data analysis and statistics and intelligent analysis, early warning, prevent [5].

Monitoring configuration interface provides a good man-machine interface, in addition to meet the monitoring function, but also to achieve energy optimization, data processing. Feed production system needs to build a unified platform, set up data centers, build network system, can grow, expand, meet the needs of future industry development.

Simulation screen display security system selenium granule pig feed the whole process and the factory monitoring is the core of the whole system, directly reflects the status of the field of technology, provides a good platform for production process [6], energy conservation, data monitoring, fault diagnosis. Selenium rich pig feed production process simulation screen as shown in figure 3 


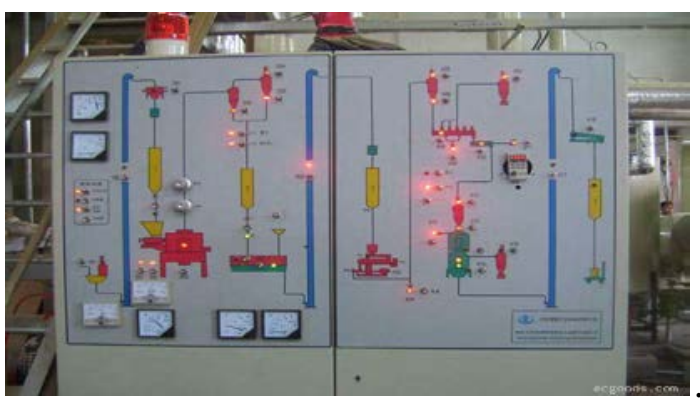

Fig. 3 simulation of selenium enriched pig feed production process.

\section{Conclusion}

The automatic control system has perfect function of selenium particles network and innovative thinking mode of pig feed production line based on the unified management of data, with the optimum allocation of equipment, energy saving advantages, improve the anti-jamming performance and control precision at the same time, the design of a new type of selenium granule pig feed crushing device solves the disadvantages of uneven grinding feed in the traditional process, make the process more precise, more perfect.

\section{Acknowledgement}

Study on the selection of agricultural and livestock products in Jilin Province, the development of electronic commerce mode and path of research by science and Technology Department of Jilin province project (project number: 20160418024FG) support.

\section{Reference}

[1] Liu Yanrong. Design of automatic batching system in feed factory [J]. electronic design Engineering works. 2012 (12)

[2] Fuzzy neural network based on improved FCM clustering algorithm for water quality of T-S Evaluation methods [D]. computer and applied chemistry, 2013 (23)

[3] Li YaFeng, Zhang XiaoNing, Wang Xin. Application Of Baffled-Submerged Bio-Film Process in the Treatment of Sewage Wastewater in a Small Town[J]. Advanced Materials Research. 2013(356). 2843-2846.

[4] Zhang Hongyu, Cheng Zongjia, Chen Yiqun, Hou Danxi, Ceng Xianhai, Zhang Guangmin, Effects of soup seagull and glucose oxidase on growth performance of Weaned Piglets[J]. feed industry.2014 (10)

[5] Zhu He. Research and design of feed production line control system based on PLC[J]. science and Technology Plaza.2013 (05) 